AperTO - Archivio Istituzionale Open Access dell'Università di Torino

Mitochondrial Targeting of Doxorubicin Eliminates Nuclear Effects Associated with Cardiotoxicity

This is a pre print version of the following article:

Original Citation:

Availability:

This version is available http://hdl.handle.net/2318/1526617

since 2015-10-17T14:09:47Z

Published version:

DOI:10.1021/acschembio.5b00268

Terms of use:

Open Access

Anyone can freely access the full text of works made available as "Open Access". Works made available under a Creative Commons license can be used according to the terms and conditions of said license. Use of all other works requires consent of the right holder (author or publisher) if not exempted from copyright protection by the applicable law. 


\section{Mitochondrial Sequestration of Doxorubicin Eliminates Nuclear Effects Associated with Cardiotoxicity}

David V. Tulumello ${ }^{\dagger, \#}$, Sae Rin Jean ${ }^{\ddagger}, \#$, Chiara Riganti ${ }^{\S}$, Liyanage $\mathrm{SU}^{\diamond}$, Schimmer $\mathrm{AD}^{\diamond}$ and Shana O. Kelley ${ }^{*} \dagger, \ddagger, \not$

${ }^{\dagger}$ Department of Biochemistry, Faculty of Medicine, ${ }^{\ddagger}$ Department of Chemistry, Faculty of Arts and Science, ${ }^{\infty}$ Department of Pharmaceutical Sciences, Leslie Dan Faculty of Pharmacy, University of Toronto, Ontario, Canada.

${ }^{\S}$ Department of Oncology, University of Torino, via Santena 5/bis, 10126 Torino, Italy

${ }^{\ominus}$ Princess Margaret Cancer Centre, Ontario Cancer Institute, University Health Network, Room 7-116, 610 University Ave, Toronto, ON, M5G 2M9, Canada

*(S.O.K.) E-mail: shana.kelley@utoronto.ca.

\#These authors contributed equally to this work.

Abstract: The highly effective anticancer agent doxorubicin (Dox), is a frontline drug used to treat a number of cancers including leukemias, ovarian, prostate, breast, and lung cancer. While Dox has a high level of activity against cancer cells, treatment is often complicated by doselimiting cardiotoxicity. For many years, this debilitating side effect is thought to originate from the drug's direct activity in the mitochondria of cardiac cells, while recent studies have shown that these are primarily downstream effect from nuclear damage. Our lab has developed a mitochondrially-targeted derivative of Dox that enables the selective study of toxicity generated by the presence of Dox in the mitochondria of $\mathrm{H} 9 \mathrm{c} 2$ rat cardiomyocytes. We demonstrate that mitochondria-targeted doxorubicin (mtDox) lacks any direct nuclear effects, which allows the cardiomyocytes to undergo mitochondrial biogenesis. This recovery response compensates for the mitotoxic effects of Dox and prevents cell death in cardiomyocytes. In accordance with these findings, cardiac toxicity was only observed in Dox but not mtDox treated mice. This study 
provides valuable insight into the development of methods to effectively limit the debilitating cardiotoxic effects of Dox, and potentially of other chemotherapeutics that exert effects on multiple subcellular organelles..

\section{Introduction}

Anthracyclines such as doxorubicin (Dox) are highly effective chemotherapeutic agents in widespread use for treatment of a variety of cancers including leukemias, ovarian, breast and lung cancer. (1) Since the initial discovery and development of Dox as an antitumor agent in the 1960s, a variety of cellular mechanisms of its potent cytotoxic activity have been identified. (2) The primary mode of action is thought to be through binding at the topoisomerase II (TopoII)DNA interface to form covalent TopoII-DNA cleavage complexes, ultimately disrupting DNA replication and transcription. $(3,4)$ Through this process, the formation of TopoII cleavage complexes result in the generation of nuclear DNA (nDNA) double stranded breaks (DSBs) leading to the induction of apoptosis. In addition, Dox is a redox active compound that can cause cell death by generating reactive oxygen species (ROS), which can lead to lipid peroxidation and oxidative DNA lesions.

While Dox is an extremely potent anticancer agent, its clinical use is often complicated by dose-limiting cardiotoxicity, causing congestive heart failures in $20 \%$ of patients.(5) At the cellular level, anthracycline-induced cardiomyopathy has long been associated with mitochondrial dysfunction - in particular the generation of high levels of ROS due to redox cycling of Dox at complex I of the electron transport chain (ETC).(6, 7) Alterations in iron and calcium homeostasis have also been identified as contributing factors. Furthermore, TopoII has also been observed in mitochondria(8), although the contributions of mitochondrial 
topoisomerase inhibition to cardiotoxicity have only just begun to be explored.(9) Recently, nDNA damage induced by interaction of TopoII was shown to be responsible for the cardiotoxic effects of Dox in mouse models. $(10,11)$ Dox-induced mitochondrial dysfunction may be primarily dependent upon changes in the mitochondrial transcriptome resulting from nDNA damage rather than the direct mitotoxic effects(10), consistent with previous findings linking Dox-induced cardiotoxicity to a suppression of mitochondrial biogenesis response resulting from a decrease in nuclear-encoded members of the mitochondrial transcriptome.(12) Under many environmental stresses such as exercise or oxidative damage, cardiac cells are able to adjust to these circumstances by simply generating new, functional mitochondria through the process of mitochondrial biogenesis.(13) However, in the presence of nDNA damage upon Dox treatment, mitochondrial biogenesis is impaired resulting in the death of cardiomyocytes. (12) Organellespecific contributions of Dox toxicity have yet to be fully dissected, largely due to the lack of available tools for selectively targeting drugs to a single subcellular compartment until recent years.

Aside from anthracyclines, mitochondrial dysfunction has also been associated with the mechanism of action for other chemotherapeutic agents such as cisplatin (14), which have been exploited in the development of new cancer therapeutics. (15-17) The susceptibility of cancer cells to mitochondrial disruption is due to metabolic changes in cancer cells associated with high rates of glycolysis, which does not occur in cells belonging to other tissues such as the heart. \{Fulda, $2010 \# 29\}$ (ref) While the therapeutic potential of harnessing the mitochondrial disruptive effects of Dox has been contemplated for over a decade (18), only recently have specific molecular targeting strategies been employed to deliver Dox to mitochondria. Such strategies include liposome encapsulation (19), chemical conjugation of mitochondrial targeting 
moieties $(20,21)$ chemical modification (22), and nanoparticle encapsulation (23), all of which have demonstrated the capacity to induce cell death in cancer cells, validating this therapeutic strategy. Particularly promising is the ability of these targeted compounds to overcome clinically relevant resistance mechanisms that exist outside of mitochondria.(20-22) Previous work has demonstrated that mitochondria-targeted Dox (mtDox, see Figure 1A for structure) is able to inhibit TopoII activity in vitro, leading to the induction of apoptotic cell death in cancer cells, while evading resistance mechanisms such as the upregulation of Pgp efflux pumps in the cellular membrane. (20) This phenomenon has been observed for a number of targeted anticancer agents and is likely the result of direct mitochondrial sequestration.(24)

We propose that by leveraging this targeting approach, we can probe and eliminate the aforementioned nuclear effects associated with Dox-induced cardiotoxicity.(20) As mtDox would only induce mtDNA damage and not nDNA, a mitochondrial biogenesis response can be induced to rescue $\mathrm{H} 9 \mathrm{c} 2$ rat cardiomyocytes from cell death.(25) Upon treatment of $\mathrm{H} 9 \mathrm{c} 2$ cells with mtDox, mitochondrial biogenesis was initiated, leading to a reduction or complete elimination of cellular dysfunctions linked to chronic anthracycline-induced cardiomyopathy. These findings demonstrate that mitochondrial targeting is a promising way to reduce the cardiotoxic nuclear effects in Dox-treated cardiomyocytes. This study also highlights the potential use of subcellular targeting to probe the organelle-specific contributions of secondary toxicity associated with drug treatment.

\section{Methods and Materials}

Cell Culture. A2780 and H9c2 (2-1) rat cardiomyocytes were obtained from ATCC. A2780 cell were cultured in RPMI-160 media (Sigma-Aldrich, St. Louis) supplemented with 10\% (v/v) fetal bovine serum (FBS, Life Technologies). H9c2 and 143B cells were cultured in DMEM (Life 
Technologies) supplemented with 10\% (v/v) FBS and containing 4.5 g/L D-Glucose and 110 mg/L sodium pyruvate. 143B $\rho^{\circ}$ cells (donated by Douglas Wallace, University of California, Irvine) were grown in DMEM (Life Technologies) supplemented with 10\% (v/v) FBS and containing $4.5 \mathrm{~g} / \mathrm{L} \mathrm{D}$-Glucose, $5 \mathrm{mg} / \mathrm{mL}$ uridine, and $100 \mathrm{mM}$ sodium pyruvate. All cells lines were grown at $37{ }^{\circ} \mathrm{C}$ with $5 \% \mathrm{CO}_{2}$ in $\mathrm{T} 75 \mathrm{~cm}^{2}$ flask with vented caps (Sarstedt).

Peptide and drug conjugate synthesis and characterization. Solid-phase synthesis, doxorubicin-peptide conjugation and purification were performed as briefly described.(ref) Briefly, peptides were synthesized using standard FMOC chemistry, succinic anhydride was then conjugated to the N-terminus of the peptide while on resin. The peptides were cleaved from resin, precipitated in ether, and lyophilized. Doxorubicin was then conjugated to the peptide in a 2:1 molar ratio, using HBTU (3 equiv) and DIPEA (6 equiv.) in DMF overnight. The doxorubicinpeptide conjugate was then purified using RP-HPLC on a preparative C18 column. The identity of the final conjugate was confirmed using electrospray ionization mass spectrometry, and purity was determined to be $>95 \%$ using analytical RP-HPLC on a C18 column with a H2O-acetonitlre ( $0.1 \%$ TFA) gradient. Dox and mtDox concentrations were determined by absorbance in $\mathrm{ddH} 2 \mathrm{O}$ at $488 \mathrm{~nm}$ using a molecular extinction coefficient of $11500 \mathrm{M}^{-1} \mathrm{~cm}^{-1}$.

Subcellular localization and DNA staining. Cells were seeded at a density of 25000 cells/well (A2780 cells) or 12500 cells/well (H9c2) in 8-well slides (iBidi, Germany), and grown one day in advance of experiments. The cells were treated with $8 \mu \mathrm{M}$ Dox or mtDox for $1 \mathrm{hr}$ in growth media (without FBS), and MitoTracker 633 (150 nM) (Life Technologies) or Hoechst (500 nM) were added for the final $15 \mathrm{~min}$. Cells were rinsed twice with PBS, and then fixed in $4 \%$ paraformaldehyde and $1 \%$ glutaformaldehyde in PBS for 15 min on ice. Cells were rinsed twice more with PBS, and then images in PBS using an inverted Zeiss Observer Z1 microscope. For 
DNA staining, 143B and 143B $\rho^{\circ}$ cells were plated to 50\% confluency one day prior to imaging in 8-well slides (iBidi, Germany). Cells were treated with PicoGreen (Life Technologies, fixed, and imaged under the microscope.

Quantification of subcellular uptake. H9c2 and A2780 cells were plated to $80 \%$ confluency in $\mathrm{T} 75 \mathrm{~cm}^{2}$ flask with vented caps (Sarstedt). Cells were incubated with $4 \mu \mathrm{M}$ Dox and $\mathrm{mtDox}$ for 2 hr at $37{ }^{\circ} \mathrm{C}$ with $5 \% \mathrm{CO}_{2}$ and subsequently harvested using $0.25 \%$ Trypsin-EDTA (Life Technologies). Nuclear, cytosolic, and mitochondrial fractions were isolated using Cell Fractionation Kit (MitoSciences) as per the manufacturer's instructions. $50 \mu \mathrm{L}$ of each fraction was added to $50 \mu \mathrm{L}$ of $2 \%$ triton $\mathrm{x}-100$ (BioShop) in DMSO and $100 \mu \mathrm{L}$ of $50 \%$ ethanol/0.3M hydrochloric acid in deionized water in triplicates on a white, opaque 96-well plate (Greiner BioOne) and shaken for $5 \mathrm{~min}$. The fluorescence (excitation: $490 \mathrm{~nm}$, emission: $590 \mathrm{~nm}$ ) was measured on a spectrophotometer (SpectraMax) and the percentage of drug in each subcellular fraction was then calculated.

Western blotting. H9c2 and A2780 cells were plated to $80 \%$ confluency in $775 \mathrm{~cm}^{2}$ flask with vented caps (Sarstedt). Cells were incubated with $4 \mu \mathrm{M}$ Dox and mtDox for $2 \mathrm{hr}$ at $37^{\circ} \mathrm{C}$ with $5 \%$ $\mathrm{CO}_{2}$ and subsequently harvested using $0.25 \%$ Trypsin-EDTA (Life Technologies). Following treatment, cells were rinsed twice with ice cold PBS and lysed with RIPA lysis buffer supplemented with PMSF and Protease/Phosphatise Inhibitor Cocktail (Cell signalling Technologies) for $30 \mathrm{~min}$ on ice with periodic vortexing. Cell lysates were then centrifuged at 16 $000 \mathrm{~g}$ for $10 \mathrm{~min}$ at $4{ }^{\circ} \mathrm{C}$ and the supernatant was collected. Total protein concentrations of each sample were determined using the BCA assay, and 20-40 $\mu \mathrm{g}$ of total protein were analyzed using 4-12\% gradient precast SDS-PAGE gels (Bio-Rad). Gels were transferred to PVDF membranes, and blocked in 5\% milk (v/v) in TBST buffer a minimum of 2 hours. Membranes were then 
probed overnight with primary antibodies as follows; 1:1000 anti- $\gamma \mathrm{H} 2 \mathrm{AX}$ (Abcam) in 5\% milk TBST and 1:500 glyceraldehyde 3-phosphate dehydrogenase (GAPDH, Cell Signalling Technologies) in 5\% milk TBST. Blots were rinsed 3 times with TBST and blotted with a secondary anti-rabbit IgG - HRP linked antibody (1:1000 for 2-6 hr in TBST), and bands were detected using standard chemiluminescence detection.

Measurements of ATP levels, TCA cycle activity, and ETC activity. All measurements were performed as described previously.(ref)-... see attached file

Flow cytometry measurement of cellular uptake, mitochondrial membrane potential, ROS and mitochondrial volume. Cells were seeded one day prior to treatment in 12 well flat bottom tissue culture plates (Corning, NY) at densities of 10000 per well (A2780) or 50000 per well (H9c2). All treatments were performed in culture medium without FBS. Following treatment with mtDox or Dox at $37{ }^{\circ} \mathrm{C}$, and with fluorescence probes as described for each experiment below, cells were trypsinized, rinsed twice with ice cold PBS. Cells were then stained will SYTOX Red (Life Technologies) for $10 \mathrm{~min}$ in order to exclude any dead cells from analysis, and were maintained on ice prior to analysis. Fluorescence was recorded on a BDFACSCanto flow cytometer (BD Biosciences). A minimum of 10000 cells were analyzed for each sample, and mean fluoresce values were determined. All dyes used in flow cytometer studies were chosen in order to minimize spectral overlap with fluorescence of the Doxorubicin moiety (concurrently measured in all experiments using a $488 \mathrm{~nm}$ laser excitation with wavelength filters to record emission in the range of 565-606 nm). For each dye spectral overlap with doxorubicin was assessed, and compensation values were obtained using the manufacturer's protocols in order to correct fluorescence levels. 
Comparison of live cell/fixed overall cellular uptake. Following recording of fluorescence in live cells, sampled were pelleted by centrifugation and fixed with 4\% PFA in PBS for 15 min on ice. Cells were rinsed twice with PBS and samples were recorded immediately under the same condition used prior to fixation. Test samples were also performed where cells were incubated in PBS in place of $4 \%$ PFA, but otherwise treated in the same manner. The overall medium fluorescence of these samples was reduced by $<5 \%$ between successive flow cytometry measurements.

Mitochondrial membrane potential. During the final $30 \mathrm{~min}$ of treatment, DiOC6(3) (3,3'Dihexyloxacarbocyanine Iodide) (Life Technologies) was added cells to a final concentration of $50 \mathrm{nM}$. Fluorescence of this dye was recorded using a $488 \mathrm{~nm}$ laser for excitation, and emission was recorded between $515-545 \mathrm{~nm}$.

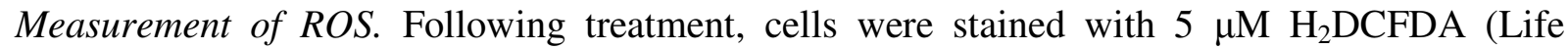
Technologies) for $30 \mathrm{~min}$ at $37 \mathrm{C}$ in PBS. Dye was then removed and cells were incubated in culture media at $37 \mathrm{C}$ for an additional $30 \mathrm{~min}$. Fluorescence of this dye was recorded using a $488 \mathrm{~nm}$ laser for excitation, and emission was recorded between $515-545 \mathrm{~nm}$.

Mitochondrial volume. During the final 30 min of treatment, MitoTracker Deep Red FM (Life Technologies) was added to cells at a final concentration of $200 \mathrm{nM}$. SYTOX Red was not added to these samples for analysis due to spectra overlap. As such, dead cells and debris were excluded during analysis solely on the basis of forward and side scattering.

Determination of mtDNA damage by qPCR. H9c2 and A2780 cells were plated to 80\% confluency in $\mathrm{T} 75 \mathrm{~cm}^{2}$ flasks with vented caps or in 6-well plates (BD). Cells were incubated with indicated concentrations of Dox or mtDox for 6 or $24 \mathrm{hr}$ at $37^{\circ} \mathrm{C}$ with $5 \% \mathrm{CO}_{2}$. Cells were then harvested using 0.25\% Trypsin-EDTA (Life Technologies), and total DNA were isolated 
using AllPrep DNA/RNA Mini Kit (Qiagen) or GenElute mammalian genomic DNA miniprep kit (Sigma) as per the manufacturer's instructions and quantified using PicoGreen (Life Technologies). An $8.9 \mathrm{~kb}$ mitochondrial segment of A2780 cells or a $3.4 \mathrm{~kb}$ mitochondrial segment of $\mathrm{H} 9 \mathrm{c} 2$ cells was amplified using the Elongase long range PCR enzyme kit (Invitrogen) as per the manufacturer's instructions and as previously described. (26) The PCR products from the treated samples were quantified in duplicates using PicoGreen (Life Technologies) and normalized to untreated sample.

Analysis of cytotoxicity. A2780 cells were seeded in 96 well plates at 10000 per well for time points $>24$ hour, and at 1000 per well for assessment of cell growth over 72 hours. H9c2 were seeded at 5000 per well for time points $>24 \mathrm{hr}, 3000$ per well for assessment of cell growth over 72 hour. Cells were treated in cell culture media without FBS for time points $>24 \mathrm{hr}$, and with FBS for $72 \mathrm{hr}$ cell viability assay.

Determination of mitochondrial protein levels. Cells were seeded in 96 well plates at density of 10000 or 5000 per well. Cells were then treated with $4 \mu \mathrm{M}$ Dox or $8 \mu \mathrm{M}$ mtDox in culture media without FBS, rinsed twice with PBS. Cell fixation, permeabilization, and ELISA was performed using the MitoBiogenesis ${ }^{\mathrm{TM}}$ In-Cell ELISA Kit (Colorimetric) (Abcam), according to manufacturer's protocol.

Measurement of mRNA levels of mitochondrial biogenesis markers by RT-qPCR. H9c2 cells were plated to $80 \%$ confluency in $\mathrm{T} 75 \mathrm{~cm}^{2}$ flask with vented caps. Cells were incubated with $1 \mu \mathrm{M}$ Dox or $8 \mu \mathrm{M}$ mtDox for $6 \mathrm{hr}$ at $37^{\circ} \mathrm{C}$ with $5 \% \mathrm{CO}_{2}$. Cells were then harvested using 0.25\% Trypsin-EDTA (Life Technologies), and total RNA were isolated using AllPrep DNA/RNA Mini Kit (Qiagen) as per the manufacturer's instructions. RNA Quantification was performed on a spectrophotometer (NanoDrop) at $260 \mathrm{~nm}$. 1-2 $\mu \mathrm{g}$ of total RNA was used for 
cDNA synthesis using gene specific primers for GAPDH, nuclear respiratory factor 1 (NRF1), and mitochondrial transcription factor A (TFAM) and SuperScript ${ }^{\circledR}$ III Reverse Transcriptase (Life Technologies) as per the manufacturer's instructions. Amplification of cDNA was performed on qPCR (Applied Biosystems) using SYBR® Green PCR Master Mix (Life Technologies) in MicroAmp optical 96-well reaction plate (Life Technologies) as per the manufacturer's instructions in duplicates.

Determination of MTD and CK level. 6-7 week old male mice were used to evaluate the MTD of Dox and mtDox. Intraperitoneal injection of $3 \mathrm{mg} / \mathrm{kg}$ of each compound was administered to 10 mice per group daily for 5 days. All mice including those of the untreated group were weighed and observed daily. 24 hours following the $5^{\text {th }}$ dose, 3 mice from each group were sacrificed and serum samples were taken. Creatine Kinase levels were measured from the blood samples following standard procedures. All mouse handling and blood chemistry work were performed by Innovative Medical Research.

\section{Results and Discussion}

mtDox uptake, localization and lack of nuclear damage in H9c2 cells.

Our lab has previously demonstrated that mtDox (Figure 1A) localizes to mitochondria of both HeLa and A2780 human cancer cell lines (20). In this study, the uptake and localization of untargeted Dox was compared to mtDox in a rat cardiomyocyte cell line (H9c2). By monitoring the inherent fluorescence of Dox, it was determined that the parent compound localizes almost entirely in the nucleus. In contrast, mtDox has a primarily mitochondrial distribution, as indicated by colocalization with a commercially available mitochondria-specific dye, MitoTracker Deep Red (Figure 1B). The subcellular uptake of Dox and mtDox was quantified by measuring the fluorescence recovered from the nucleus or mitochondria of $\mathrm{H} 9 \mathrm{c} 2$ cells post 
treatment with each compound (Figure 1C). In agreement with the microscopy images, mtDox was located almost entirely in mitochondria. Conversely, the majority of Dox was located in the nucleus with a smaller fraction localizing to mitochondria. In fact, the mitochondrial accumulation of Dox was similar to that achieved by mtDox in H9c2 cells. The levels of uptake of mtDox and Dox in the nucleus and mitochondrial were measured in A2780 cells, a human ovarian cancer cell line. In this cell line, while Dox had similar overall levels of uptake and distribution to $\mathrm{H} 9 \mathrm{c} 2$ cells, mtDox had approximately double the uptake into mitochondria in comparison to the uptake achieved in H9c2 cells.

Overall cellular uptake of both Dox and mtDox in live cells was monitored using flow cytometry. Using this technique, much lower relative levels of uptake of mtDox were observed than the subcellular fractionation studies indicated. However upon fixation of these cells with paraformaldehyde, a significant increase of fluorescence was obtained in the mtDox treated cells but not for Dox treated cells (Figure S1). As doxorubicin fluorescence is highly quenched by DNA, it is possible that mtDox localized within the mitochondrial matrix is quenched due to its interaction with mitochondrial DNA (mtDNA). To test this hypothesis, the fluorescence of mtDox in 143B $\rho^{\circ}$ cells which are depleted of mtDNA was monitored. Indeed the fluorescence of mtDox is not increased upon fixation in this cell line, but is in the parental 143B cell line which contains mtDNA. The absence of mtDNA in this cell line was confirmed by lack of PicoGreen staining of mitochondria (Figure S2). These observations serve to confirm that mtDox is localized in matrix of $\mathrm{H} 9 \mathrm{c} 2$ mitochondria where it is able to interact directly with mtDNA.

The lack of any direct nuclear effect of mtDox was also confirmed by testing for the presence or absence of nDNA DSBs. The generation of DSBs resulting from TopoII poisoning in the nucleus has previously linked to be the major source of cardiotoxic effects of Dox. In H9c2 
cells, the presence of DSBs induced by Dox was confirmed by immunoblotting for $\gamma-\mathrm{H} 2 \mathrm{AX}$ histone in the nucleus, a commonly used marker specific for nDNA lesions (Figure 1D). In contrast, no nDNA DSBs were observed for mtDox treated cells, despite its previously confirmed capacity to act as a TopoII poison. Likewise in A2780 cells, Dox, but not mtDox generated an increase of $\gamma-\mathrm{H} 2 \mathrm{AX}$ above the baseline level. Overall, in H9c2 cells mtDox has equivalent mitochondrial uptake as Dox but lacks any nuclear localization and thus direct nDNA damage. This selective activity makes mtDox an excellent tool to probe the cardiotoxic effects resulting from mitochondrial dysfunction induced by Dox in the absence of any direct nuclear damage.

\section{Comparison of mitochondrial dysfunction and time course of damage}

To detect the presence of any direct induction of mitochondrial dysfunction, the ATP levels as well as the rate of tricarboxylic acid (TCA) activity of H9c2 cells was assessed upon treatment with Dox or mtDox (Figure 2A). Both compounds resulted in similar decreases in total ATP levels. A decrease in TCA cycle activity was also observed upon exposure to Dox, and to a higher degree with mtDox. The mitochondrial metabolic consequences of Dox and mtDox treatment were further analyzed by probing the flux of electrons through various complexes of the ETC (Figure 2B). For Dox, the overall reduction of ETC activity is solely attributed to a decrease of complex I activity, owing to its ability to redox cycle specifically with this protein.(ref) In contrast, mtDox is more promiscuous in its reduction of ETC flux and affects complex I and complex II+III. Surprisingly, there was an increase in activity at complex IV compared to baseline level. 
In order to efficiently generate ATP, ETC flux maintains a highly negative inward mitochondrial membrane potential. Upon treatment with mtDox, there is a drop in the mitochondrial potential that is evident after just $30 \mathrm{~min}$ of treatment (Figure S3). This explains the decrease in overall ATP levels despite the increased proton flux generated at Complex IV. Despite the comparable mitochondrial uptake, Dox does not induce a similar potential decrease; likely due to it only has a single positive net charge.

Next, the ability of these compounds to induce mitochondria-specific damage over time was assessed. After six hours of exposure, both mtDox and Dox generated high levels of ROS in H9c2 cells (Figure 3A). While ROS levels increased over 24 hours for Dox, ROS levels of mtDox-treated cells returned to baseline level. On the contrary, in A2780 cells, there was no recovery of ROS levels following treatment with either Dox or mtDox over 24 hours.

Using a PCR based assay, the levels of mtDNA damage were measured by amplifying a large segment of the mitochondrial genome.(26) In H9c2 cells, mtDNA damage was observed after 6 hours of treatment with either Dox or mtDox. In accordance with ROS levels, no mtDNA damage above baseline was detected after 24 hours in H9c2 cells treated with mtDox, whereas cumulative mtDNA damage was observed upon treatment with Dox (Figure 3B). This recovery of DNA damage within 24 hours was once again absent in A2780 cells treated with mtDox. The time course of ROS generation and presence of mtDNA lesions demonstrate that while similar levels of mitochondrial damage initially occur upon treatment with either compound, the damage induced by mtDox in cardiomycytes does not persist at 24 hours. These results indicate that in $\mathrm{H} 9 \mathrm{c} 2$ cells, the initial mitochondrial dysfunction resulting from mtDox treatment is reverted by a compensatory mechanism, which is absent upon Dox treatment. 
$\underline{\text { Time course of cellular toxicity in cardiomyocytes versus cancer cells. }}$

Given the differences in persistence of mitochondrial damage between mtDox and Dox treated cells, we next sought to investigate how these trends would translate to overall cellular toxicity at several time points. Using a clonogenic cell viability assay, the $\mathrm{LC}_{50}$ values of Dox and mtDox at 2, 6, 24, and 72 hours were determined in H9c2 and A2780 cells. Dox induced significant cell death beginning at 24 hours, becoming increasingly toxic at 72 hours in both H9c2 and A2780 cells (Figure 4A). Despite similar levels of mitochondrial uptake in H9c2 cells, mtDox is much less toxic than Dox at 24 and 72 hour time points. At these time points which cells are able to divide, mtDox induces cell death at increasingly lower concentrations for only A2780 cells, however there is little cumulative toxicity toward cardiomyocytes. This finding suggests that nDNA damage is a key contributor toward the long-term toxicity of Dox in cardiomyocytes. In H9c2 cells, exposure to mtDox - although still inducing direct mitochondrial dysfunction and damage at levels similar to Dox- do not result in cell death. These results are consistent with the observed recovery of mitochondrial dysfunction over time in mtDox, but not Dox treated cardiomyocytes. Taken together, these results indicate that mtDox treated H9c2 cells are able to recover from mitochondrial damage in the absence of nDNA damage.

When used clinically, Dox-induced cardiotoxicity is dose-dependent and cumulative over the treatment period or for many years following.(ref) As H9c2 cells appear to recover from mtDox treatment within 24 hours, it was of interest to investigate whether reduced toxicity would be observed upon successive dosing. In order to test this, the cells were treated with $1 \mu \mathrm{M}$ of Dox, which leads to $>75 \%$ cell death over 72 hours. Upon splitting the total dose into three equal fractions and treating $\mathrm{H} 9 \mathrm{c} 2$ cells every 24 hours, the overall level of cell death remained the same (Figure 4B). This methodology was repeated with a total mtDox concentration of 16 
$\mu \mathrm{M}$. When cells were treated in a single dose, there was nearly complete cell death after 72 hours of exposure. If this same total dose was given as three separate doses every 24 hours however, $>50 \%$ of the cells were viable. This further supports the observation that $\mathrm{H} 9 \mathrm{c} 2$ cells were indeed able to recover from exposure to mtDox while Dox treated cells were susceptible to cumulative damage.

$\underline{m t D o x}$ treatment induces mitochondrial biogenesis in cardiomyocytes

One mechanism by which cells compensate for damaged mitochondria is by producing new, healthy mitochondria through mitochondrial biogenesis. In order to determine if this pathway is an important mechanism for reducing mtDox toxicity toward cardiomyocytes, changes in total mitochondria were monitored following 6 and 24 hours exposure to the two compounds (Figure 5A). Firstly, the mitochondrial volume was measured by staining drug treated cells with MitoTracker Deep Red. In mtDox treated cells, there is a significant increase in mitochondrial volume compared to untreated cells. Despite Dox treated cells displaying similar level of mitochondrial dysfunction to mtDox, there was no increase in mitochondrial volume. While an increase in number of mitochondria may compensate for the mitochondrial dysfunction, damaged mitochondria may also be removed through mitophagy. Indeed by inhibiting this pathway using 3-methyladanine, further increases in mitochondrial volume were observed (Figure S4). This indicates that there is some fraction of damaged mitochondria that are cleared by mitophagy concurrently with the increase in this organelle through mitochondrial biogenesis.

In order to further validate these changes in mitochondrial number, levels of mitochondrial proteins were evaluated. Using an ELISA based assay, the relative levels of nuclear encoded (SDH-A) and mtDNA encoded (COXI) mitochondrial proteins were measured 
upon drug treatment since mitochondrial biogenesis requires the transcription and translation of both nDNA and mtDNA. Similar to what was observed for mitochondrial volume, mitochondrial protein levels increased in mtDox, but not Dox treated cells after 6 hours of exposure. Interestingly, the levels of both the mtDNA and nDNA encoded proteins were similar. This indicates that the lack of mitochondrial biogenesis induced by Dox treatment is not solely the consequence of mitochondrial dysfunction but is also dependent on the functional state of nDNA.

In mammalian cells, an increase in ROS serves as a signal of mitochondrial stress to the nucleus. In response to this signal, transcription factors such as NRF1 and TFAM are upregulated to aid in the process of mitochondrial biogenesis.(Deblois ref). Therefore, the transcript levels of NRF1 and TFAM of Dox and mtDox treated H9c2 cells were quantified. While only NRF1 levels increased significantly compared to untreated with mtDox treated cells, both NRF1 and TFAM levels were 5-fold higher in mtDox treated cells compared to Dox. Taken together, these findings demonstrate the ability of mtDox to induce mitochondrial biogenesis in cardiomyocytes to ensure their survival while Dox, in the presence of nDNA damage, are unable to recover from mitochondrial dysfunction due to impaired mitochondrial biogenesis.

\section{$\underline{\text { Systemic and cardiac-specific toxicity in vivo is not observed with mtDox administration }}$}

Finally, the overall in vivo toxicity in mice treated with Dox or mtDox was evaluated by determining MTD (maximum tolerated dose). At the dosing regimen of $3 \mathrm{mg} / \mathrm{kg} /$ day for 5 days, Dox and mtDox treated mice maintained less than $10 \%$ weight loss (Figure 6A). Next, CK (ereatine kinase) levels were measured in serum samples from treated mice as a marker for cardiac toxicity. Interestingly, only Dox treated mice had significant increase in CK levels compared to untreated mice (Figure 6B). In addition, Dox treated mice had CK levels $\sim 8$ fold 
higher than that of untreated and $\sim 4$ fold higher than that of mtDox treated mice. These findings demonstrate that mtDox does not induce systemic or cardiac-specific toxicity in mice in accordance with the in cellulo data.

\section{Conclusions}

Given the off-target toxicity of Dox, it is an attractive proposition to produce derivatives of this drug with specific subcellular activity in an effort to maintain toxicity against cancer cells but reduce unwanted secondary toxicity in normal cells. The nuclear effects of Dox were eliminated through mitochondrial sequestration, allowing us to selectively study the importance of nDNA damage in cardiomyocyte toxicity upon drug treatment. In this study, we demonstrated that mitochondria-targeting significantly reduced toxicity of Dox toward cardiomyocytes, in accordance with recent reports in animal models that show nDNA damage plays a major role in anthracycline-induced cardiotoxicity. Although short-term mitochondrial dysfunction was observed in cardiomyocytes upon treatment with mtDOX, these effects were compensated for by mitochondrial biogenesis and do not persist at treatment concentrations where this compound exhibits anticancer activity. As demonstrated previously by the ability of mtDox to evade resistance mechanisms, combined with its reduced toxicity in cardiomyocytes and mice, mitochondria-targeting presents a promising strategy for the development of novel chemotherapeutics. Thus, subcellular targeting of Dox enables us to simultaneously study and eliminate the key cellular mechanism of cardiotoxicity. Furthermore, targeted compounds are powerful tools that can be used to deconvolute the organelle-specific effects for drugs that possess a wide range of cellular activity. A greater understanding of these cellular mechanisms will allow for the development of new pharmaceutical intervention strategies that have the ability to eliminate highly undesirable off-target effects while maintaining potent anticancer activity. 


\section{References}

Other references:

decrease in mito potential activates nuc signalling pathway and then p53(27)

1. Tacar, O., Sriamornsak, P., and Dass, C. R. (2013) Doxorubicin: an update on anticancer molecular action, toxicity and novel drug delivery systems, J Pharm Pharmacol 65, 157170.

2. Weiss, R. B. (1992) The anthracyclines: will we ever find a better doxorubicin?, Semin Oncol 19, 670-686.

3. Nitiss, J. L. (2009) Targeting DNA topoisomerase II in cancer chemotherapy, Nat Rev Cancer 9, 338-350.

4. Pommier, Y. (2013) Drugging topoisomerases: lessons and challenges, ACS Chem Biol 8, 82-95.

5. Minotti, G., Menna, P., Salvatorelli, E., Cairo, G., and Gianni, L. (2004) Anthracyclines: molecular advances and pharmacologic developments in antitumor activity and cardiotoxicity, Pharmacol Rev 56, 185-229.

6. Singal, P. K., and Iliskovic, N. (1998) Doxorubicin-induced cardiomyopathy, $N$ Engl J Med 339, 900-905.

7. Berthiaume, J. M., and Wallace, K. B. (2007) Adriamycin-induced oxidative mitochondrial cardiotoxicity, Cell Biol Toxicol 23, 15-25.

8. Low, R. L., Orton, S., and Friedman, D. B. (2003) A truncated form of DNA topoisomerase IIbeta associates with the mtDNA genome in mammalian mitochondria, Eur J Biochem 270, 4173-4186.

9. Khiati, S., Dalla Rosa, I., Sourbier, C., Ma, X., Rao, V. A., Neckers, L. M., Zhang, H., and Pommier, Y. (2014) Mitochondrial topoisomerase I (Top1mt) is a novel limiting factor of doxorubicin cardiotoxicity, Clin Cancer Res.

10. Zhang, S., Liu, X., Bawa-Khalfe, T., Lu, L. S., Lyu, Y. L., Liu, L. F., and Yeh, E. T. (2012) Identification of the molecular basis of doxorubicin-induced cardiotoxicity, Nat Med 18, 1639-1642.

11. Sawyer, D. B. (2013) Anthracyclines and heart failure, N Engl J Med 368, 1154-1156.

12. Suliman, H. B., Carraway, M. S., Ali, A. S., Reynolds, C. M., Welty-Wolf, K. E., and Piantadosi, C. A. (2007) The CO/HO system reverses inhibition of mitochondrial biogenesis and prevents murine doxorubicin cardiomyopathy, J Clin Invest 117, 37303741 .

13. Ventura-Clapier, R., Garnier, A., and Veksler, V. (2008) Transcriptional control of mitochondrial biogenesis: the central role of PGC-1alpha, Cardiovascular research 79, 208-217.

14. Cullen, K. J., Yang, Z., Schumaker, L., and Guo, Z. (2007) Mitochondria as a critical target of the chemotheraputic agent cisplatin in head and neck cancer, J Bioenerg Biomembr 39, 43-50.

15. Zhang, X., Fryknas, M., Hernlund, E., Fayad, W., De Milito, A., Olofsson, M. H., Gogvadze, V., Dang, L., Pahlman, S., Schughart, L. A., Rickardson, L., D'Arcy, P., 
Gullbo, J., Nygren, P., Larsson, R., and Linder, S. (2014) Induction of mitochondrial dysfunction as a strategy for targeting tumour cells in metabolically compromised microenvironments, Nat Commun 5, 3295.

16. Skrtic, M., Sriskanthadevan, S., Jhas, B., Gebbia, M., Wang, X., Wang, Z., Hurren, R., Jitkova, Y., Gronda, M., Maclean, N., Lai, C. K., Eberhard, Y., Bartoszko, J., Spagnuolo, P., Rutledge, A. C., Datti, A., Ketela, T., Moffat, J., Robinson, B. H., Cameron, J. H., Wrana, J., Eaves, C. J., Minden, M. D., Wang, J. C., Dick, J. E., Humphries, K., Nislow, C., Giaever, G., and Schimmer, A. D. (2011) Inhibition of mitochondrial translation as a therapeutic strategy for human acute myeloid leukemia, Cancer Cell 20, 674-688.

17. Fonseca, S. B., Pereira, M. P., Mourtada, R., Gronda, M., Horton, K. L., Hurren, R., Minden, M. D., Schimmer, A. D., and Kelley, S. O. (2011) Rerouting chlorambucil to mitochondria combats drug deactivation and resistance in cancer cells, Chem Biol 18, 445-453.

18. Jung, K., and Reszka, R. (2001) Mitochondria as subcellular targets for clinically useful anthracyclines, Adv Drug Deliv Rev 49, 87-105.

19. Malhi, S. S., Budhiraja, A., Arora, S., Chaudhari, K. R., Nepali, K., Kumar, R., Sohi, H., and Murthy, R. S. (2012) Intracellular delivery of redox cycler-doxorubicin to the mitochondria of cancer cell by folate receptor targeted mitocancerotropic liposomes, Int $J$ Pharm 432, 63-74.

20. Chamberlain, G. R., Tulumello, D. V., and Kelley, S. O. (2013) Targeted Delivery of Doxorubicin to Mitochondria, ACS Chem Biol 8, 1389-1395.

21. Han, M., Vakili, M. R., Soleymani Abyaneh, H., Molavi, O., Lai, R., and Lavasanifar, A. (2014) Mitochondrial Delivery of Doxorubicin via Triphenylphosphine Modification for Overcoming Drug Resistance in MDA-MB-435/DOX Cells, Mol Pharm.

22. Riganti, C., Rolando, B., Kopecka, J., Campia, I., Chegaev, K., Lazzarato, L., Federico, A., Fruttero, R., and Ghigo, D. (2013) Mitochondrial-targeting nitrooxy-doxorubicin: a new approach to overcome drug resistance, Mol Pharm 10, 161-174.

23. Theodossiou, T. A., Sideratou, Z., Katsarou, M. E., and Tsiourvas, D. (2013) Mitochondrial delivery of doxorubicin by triphenylphosphonium-functionalized hyperbranched nanocarriers results in rapid and severe cytotoxicity, Pharm Res 30, 28322842.

24. Rin Jean, S., Tulumello, D. V., Wisnovsky, S. P., Lei, E. K., Pereira, M. P., and Kelley, S. O. (2014) Molecular vehicles for mitochondrial chemical biology and drug delivery, ACS Chem Biol 9, 323-333.

25. Horton, K. L., Stewart, K. M., Fonseca, S. B., Guo, Q., and Kelley, S. O. (2008) Mitochondria-penetrating peptides, Chem Biol 15, 375-382.

26. Santos, J. H., Meyer, J. N., Mandavilli, B. S., and Van Houten, B. (2006) Quantitative PCR-based measurement of nuclear and mitochondrial DNA damage and repair in mammalian cells, Methods in molecular biology (Clifton, N.J.) 314, 183-199.

27. Liu, J., Mao, W., Ding, B., and Liang, C. S. (2008) ERKs/p53 signal transduction pathway is involved in doxorubicin-induced apoptosis in H9c2 cells and cardiomyocytes, Am J Physiol Heart Circ Physiol 295, H1956-1965. 


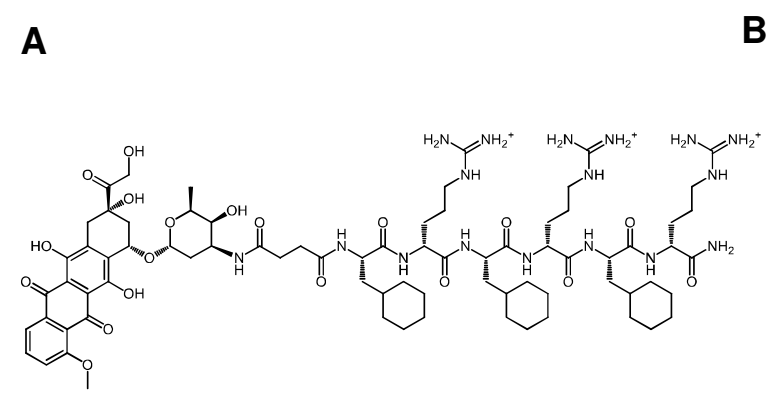

B

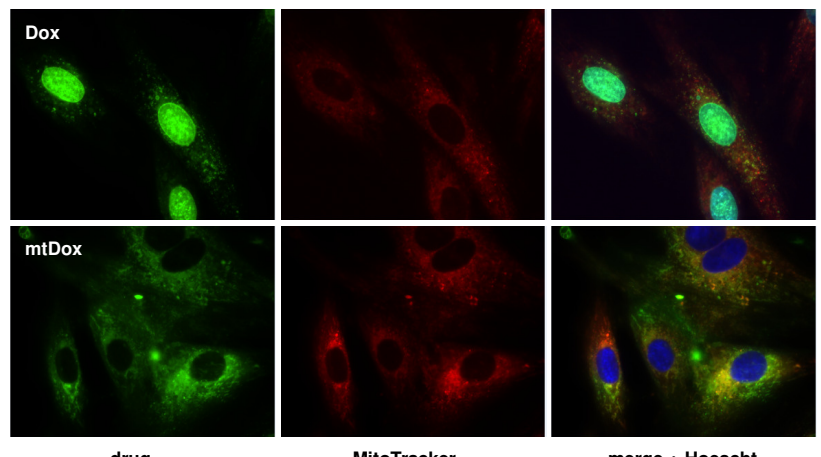

C

D

drug

MitoTracker

merge + Hoescht
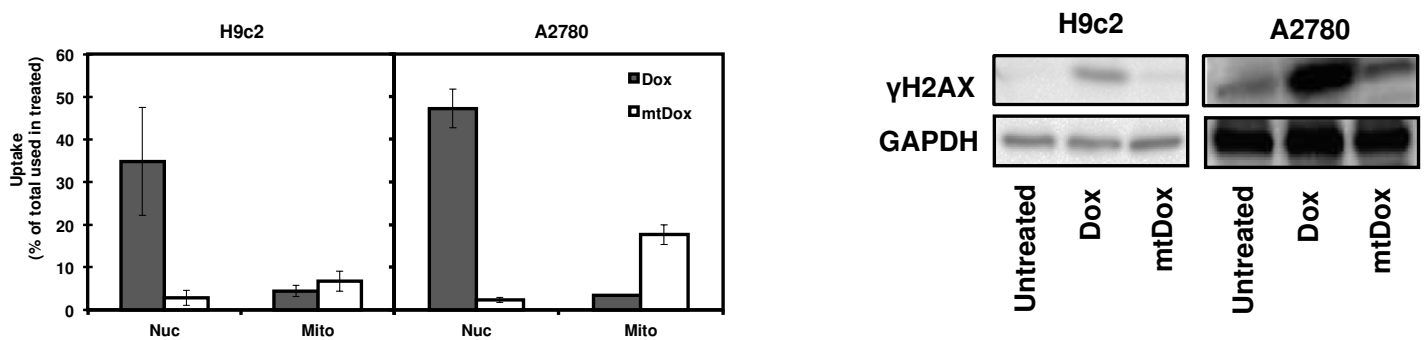

Figure 1. Comparison of uptake, subcellular localization and induction of nuclear damage. (A) The chemical structure of doxorubicin conjugated to a mitochondria-penetrating peptide (mtDox) (B) Subcellular localization of Dox (top row) and mtDox (bottom row) in H9c2 cardiomyocytes. Intrinsic Dox fluorescence (green channel) demonstrates nuclear staining, as indicated by colocalization with Hoescht (blue channel in merge). mtDox accumulates in mitochondria as indicated by colocalization with MitoTracker Deep Red (red channel). (C) Quantification of relative levels of accumulation of $4 \mu \mathrm{M}$ Dox or mtDox in nuclear or mitochondrial fractions of H9c2 (left panel) or A2780 (right panel). Uptake is shown as a percentage of total amount of compound used to treat cells. Mean values are plotted and error bars represent SEM. (D) Western blot of $\gamma$-H2AX and GAPDH (loading control) of H9c2 (left panel) of A2780 (right panel) cells treated with Dox ( $4 \mu \mathrm{M}$ in A2780 cells, $4 \mu \mathrm{M}$ in H9c2 cells) or mtDox ( $4 \mu \mathrm{M}$ in A2780 cells, $8 \mu \mathrm{M}$ in H9c2 cells) for 2 hours. 

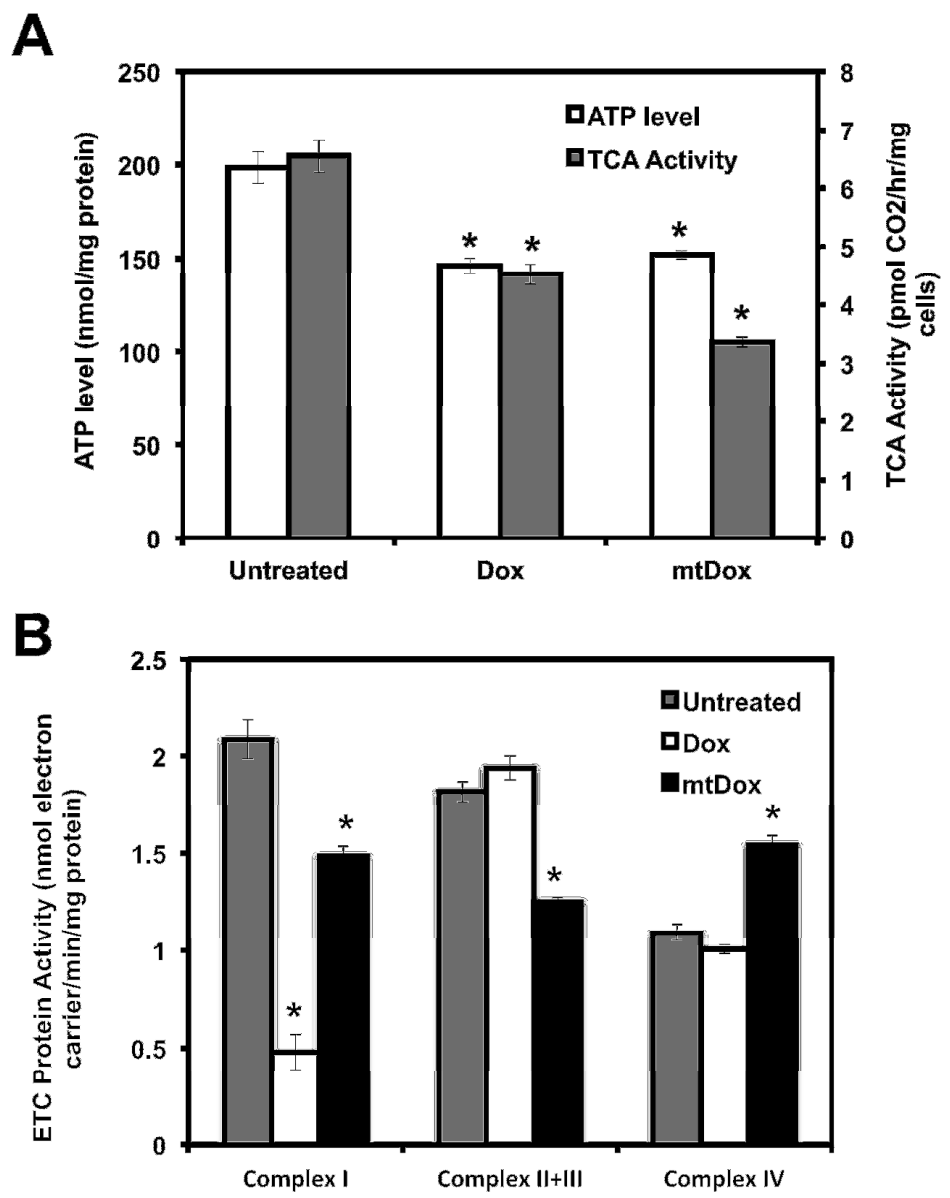

Figure 2. Mitochondrial dysfunction induced by drug treatment. (A) Measurement of ATP level and TCA activity upon treatment of H9c2 cells with $5 \mu \mathrm{M}$ of Dox or mtDox. (B) Determination of Complex I-IV activity of ETC in H9c2 cells treated with $5 \mu \mathrm{M}$ of Dox or mtDox. Mean values are plotted for all experiments and error bars represent SEM. Student's t test, versus untreated control $* p<0.05$. 
A
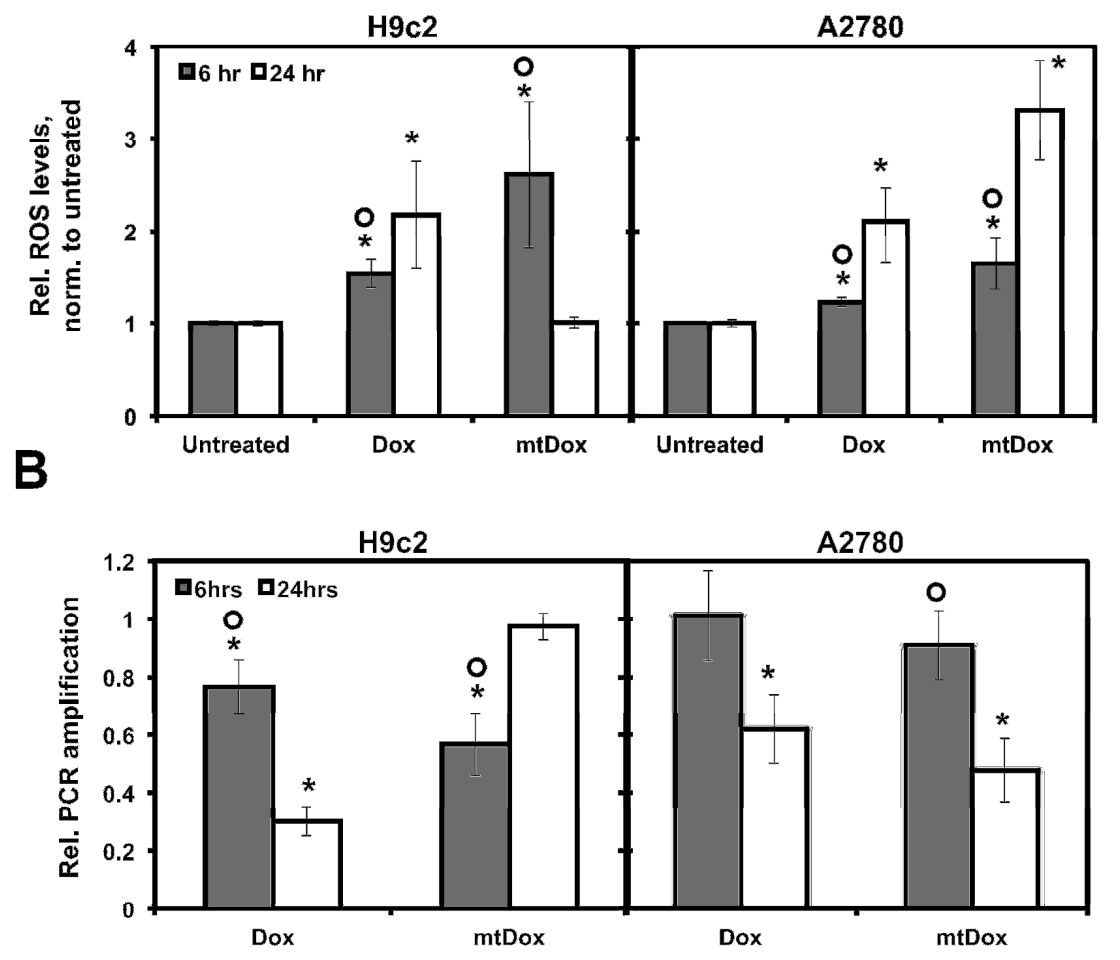

Figure 3. Induction and recovery of mitochondrial specific damage. (A) Relative levels of total cellular ROS following 6 or 24 hour treatment of H9c2 (left panel) or A2780 (right panel) with Dox or mtDox. Total fluorescence values of a ROS sensitive dye were normalized to untreated controls. (B) Relative PCR amplification of mtDNA damage following 6 or 24 hour treatment of H9c2 (left panel) or A2780 (right panel) with Dox or mtDox normalized to untreated cells. Mean values are plotted for all experiments and error bars represent SEM. Student's t test, versus untreated control $* p<0.05$, versus 24 hour ${ }^{\circ} p<0.05$. 


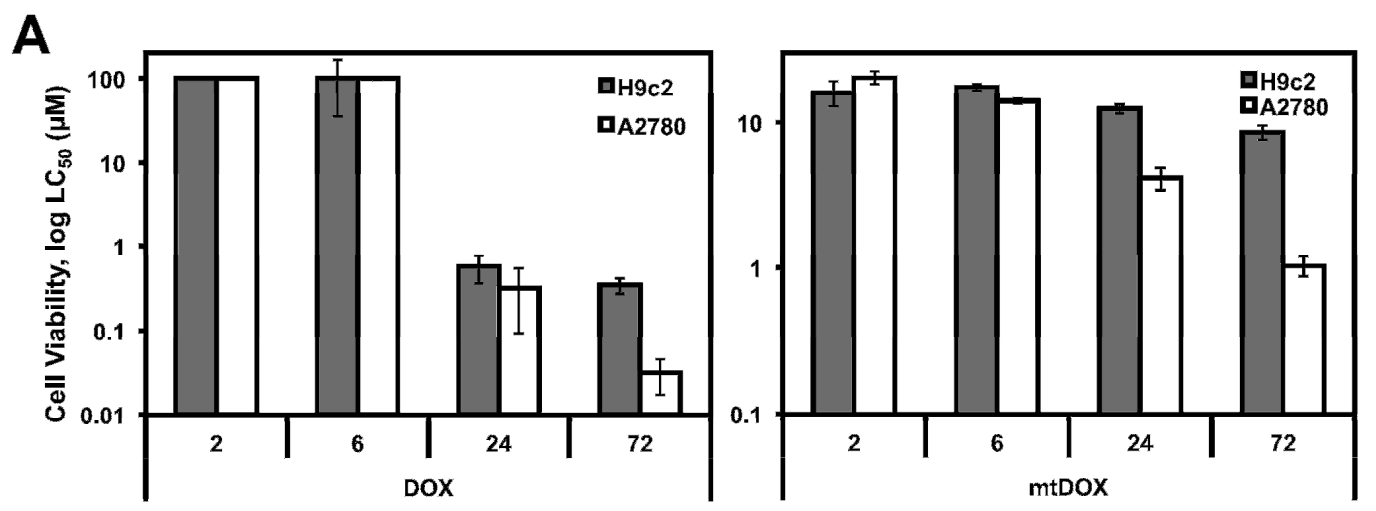

B

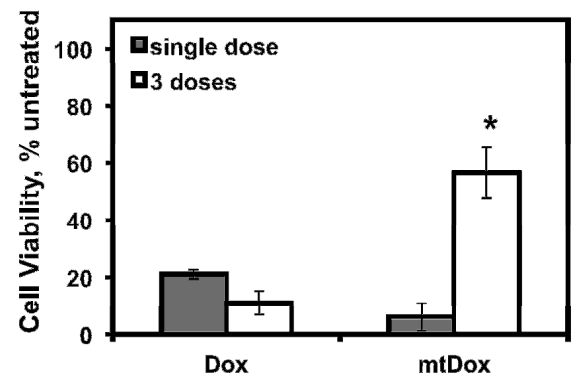

Figure 4. Time course of cell death of $H 9 c 2$ versus $A 2780$. (A) $\mathrm{LC}_{50}$ values following treatment with Dox (left panel) or mtDox (right panel) in H9c2 and A2780 cells. (B) Comparison of cell viability following single or 3 dispersed dosing over 72 hours with Dox or mtDox in H9c2 cells. Mean values are plotted for all experiments and error bars represent SEM. Student's t test, versus single dose $* p<0.05$. 


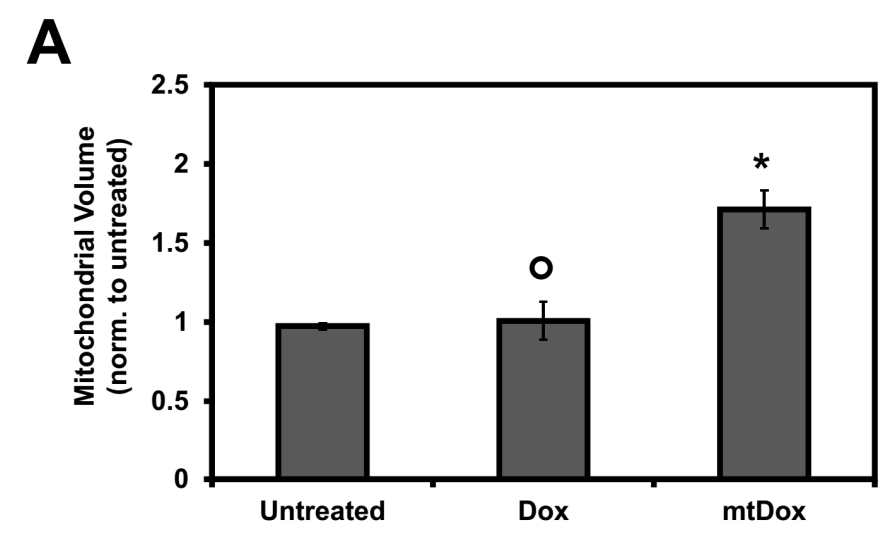

B
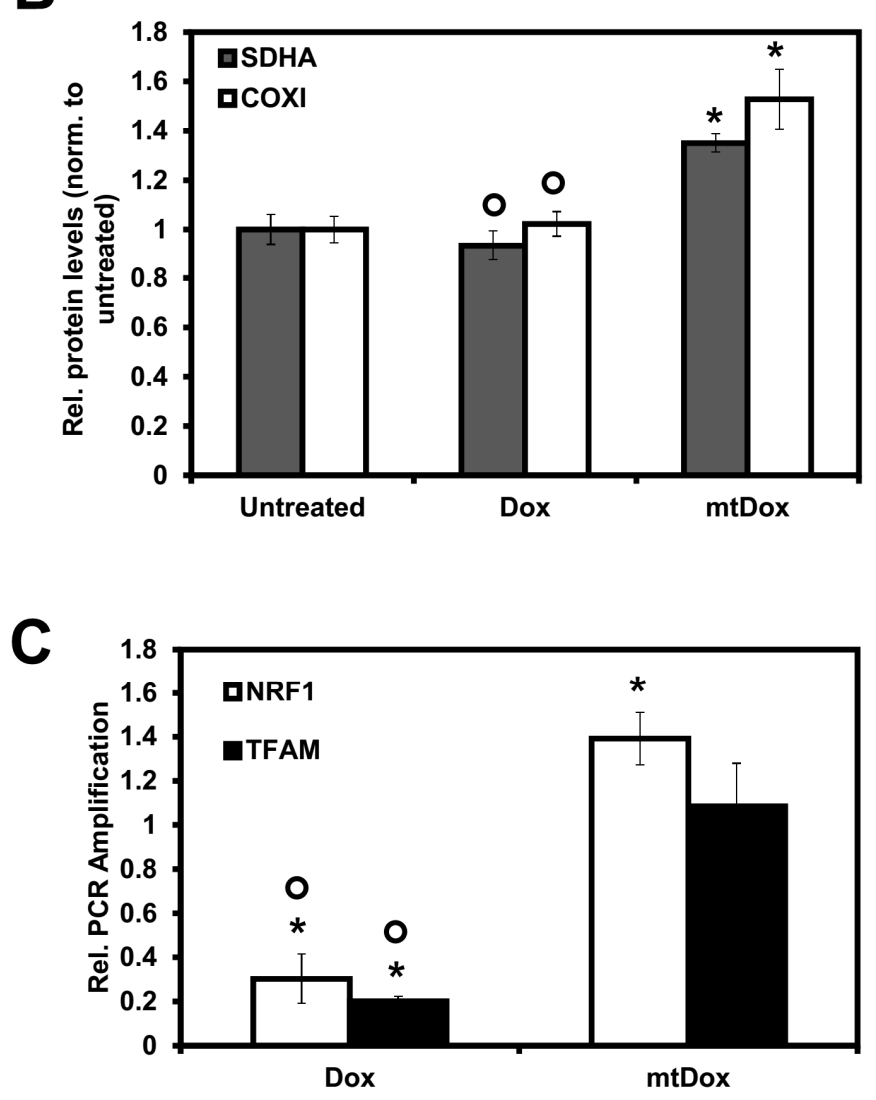

Figure 5. Induction of mitochondrial biogenesis upon mtDox treatment. (A) Measurement of mitochondrial volume following 6 hour treatment with Dox or mtDox. (B) Comparison of mitochondrial protein levels by ELISA immunological assay following a 6 hour treatment with Dox or mtDox. (C) Quantification of transcript levels by RT-qPCR following 6 hour treatment with Dox or mtDox, normalized to untreated control. All of these experiments were analyzed by Mean values are plotted for all experiments and error bars represent SEM. Student's t test, versus untreated control $* p<0.05$, versus $\operatorname{mtDox}{ }^{\circ} p<0.05$. 
A

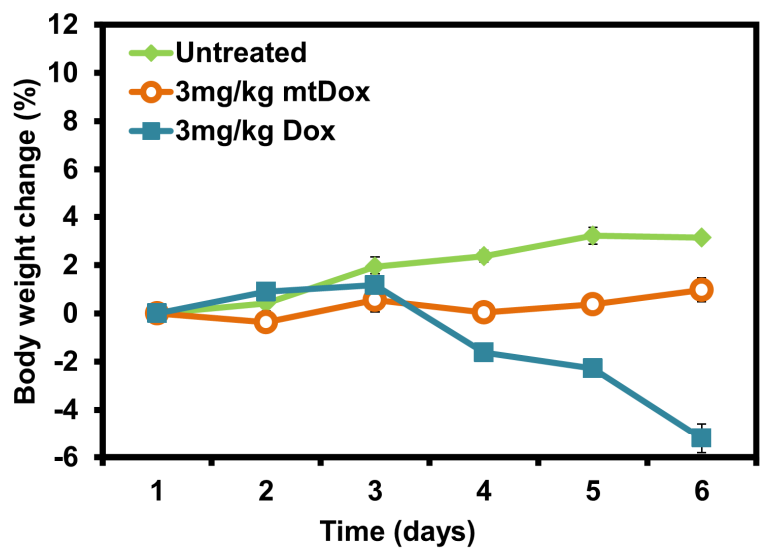

B

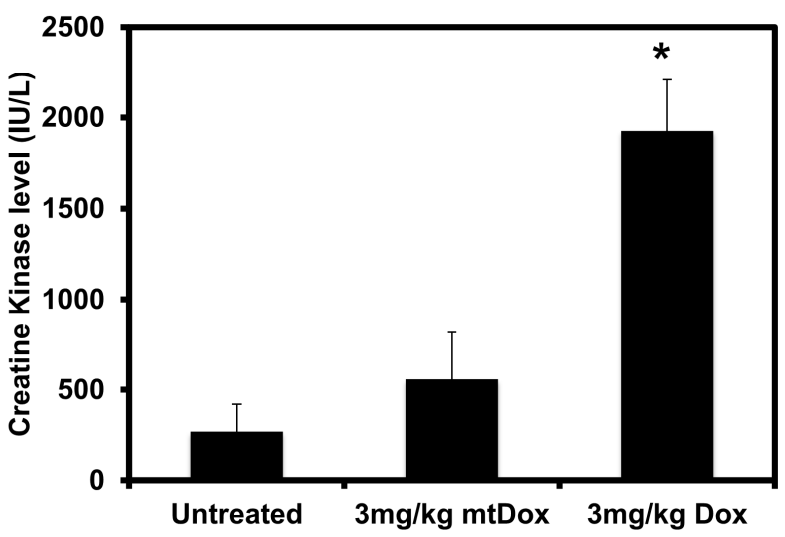

Figure 6. Evaluation of general and cardiac-specific toxicity. A) Determination of MTD (maximum tolerated dose). Compounds were administered intraperitoneally daily for 5 days ( $\mathrm{n}=$ 5-10). MTD values of each compound was estimated based on the threshold at which all of the mice survived with a body weight loss $<10 \%$. B) Measurement of CK (creatine kinase) level in treated mice. Serum was collected 24 hours after the $5^{\text {th }}$ dose and $(n=3)$. Mean values are plotted for all experiments and error bars represent SEM. Student's t test, versus untreated control $* p<0.05$. 
TOC:

Dox
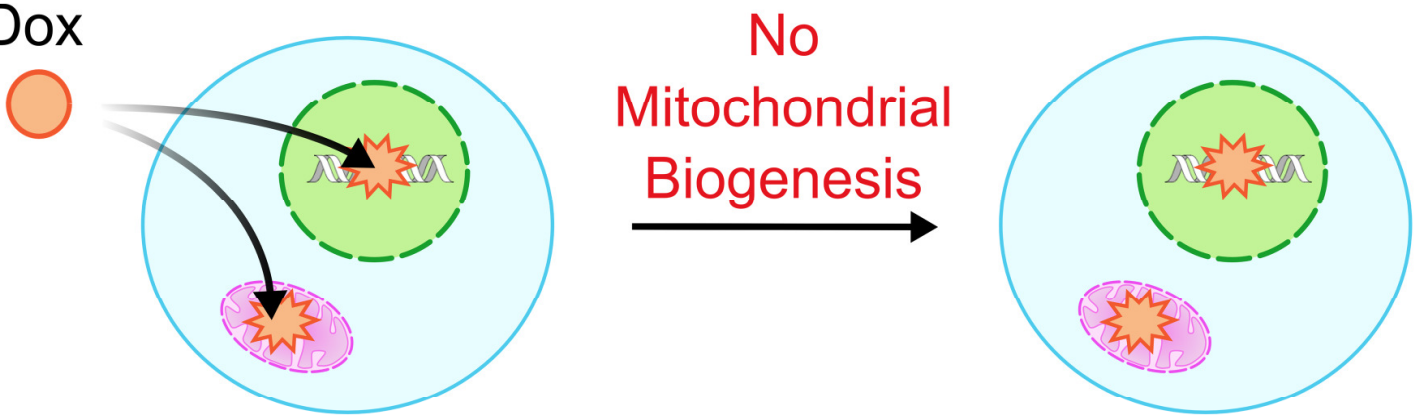

Cardiomyocyte Death
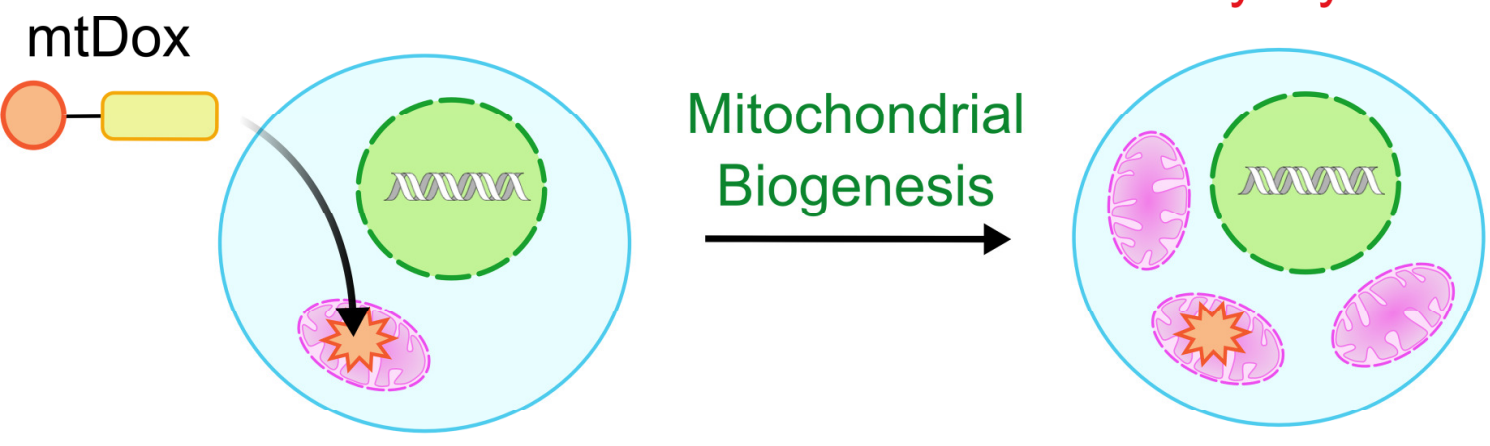

Cardiomyocyte Survival

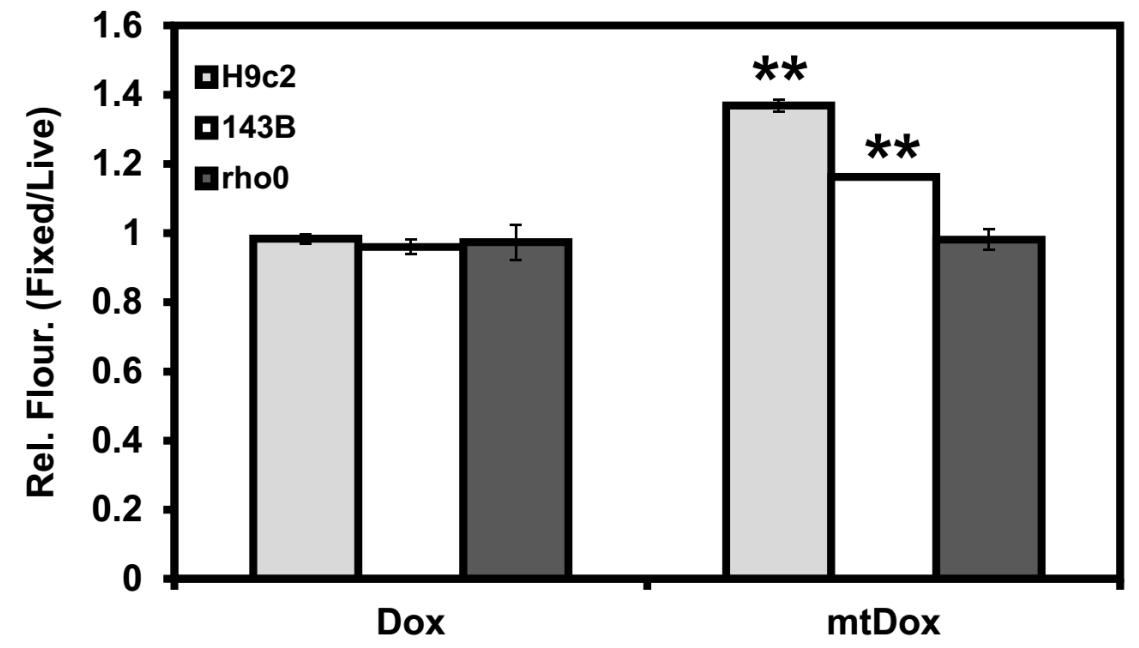

Figure S1. Ratio of total cellular fluorescence prior to and following cell fixation with paraformaldyehyde. Results are shown for H9c2 cardiomyocytes, 143B cells and 143B rho0 cells (mtDNA has been depleted by chronic treatment with ethidium bromide). 

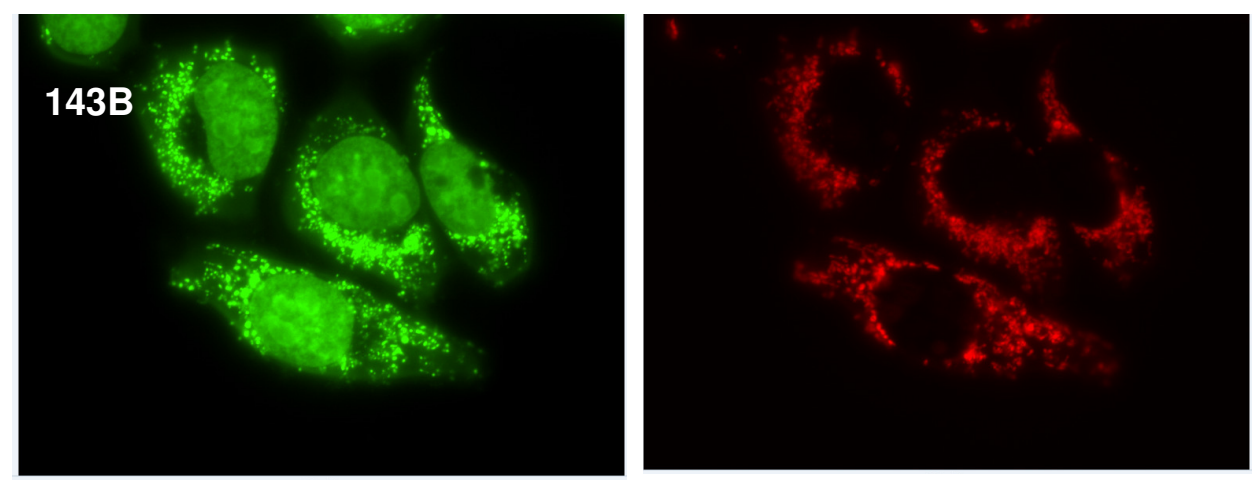

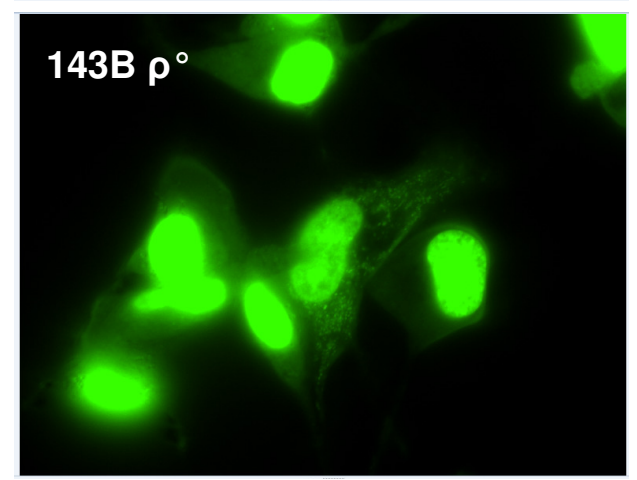

PicoGreen

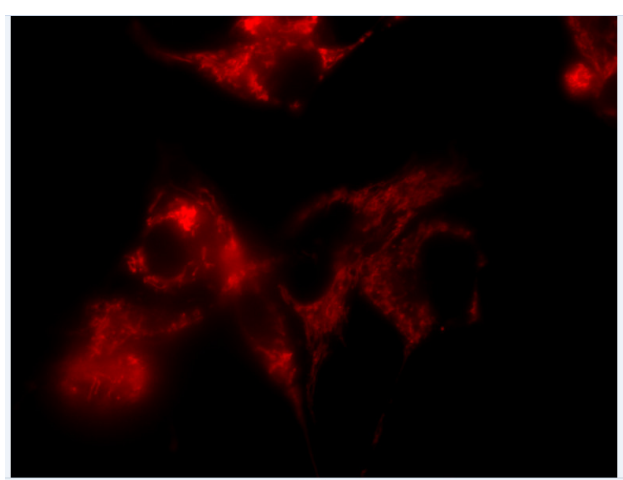

MitoTracker

Figure S2. Comparison of mtDNA content by fluorescence microscopy using PicoGreen dye.

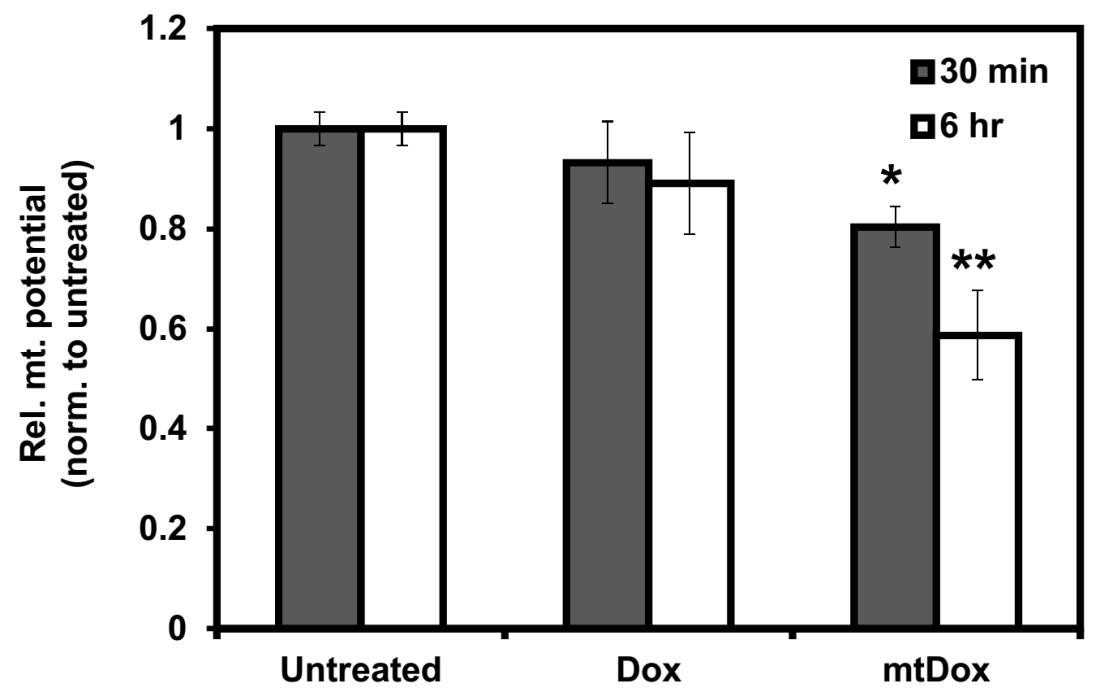

Figure S3. Relative mitochondrial potential following drug treatment. Levels of uptake of a mitochondrial potential sensitive dye were normalized to untreated controls. 